\title{
A non-redundant role for OX40 in the competitive fitness of Treg in response to IL-2
}

\author{
Silvia Piconese ${ }^{* 1}$, Paola Pittoni ${ }^{* 1}$, Alessia Burocchi ${ }^{1}$, Andrea Gorzanelli ${ }^{1}$, \\ Alessandra Car $\grave{e}^{2}$, Claudio Tripodo ${ }^{3}$ and Mario P. Colombo ${ }^{1}$ \\ ${ }^{1}$ Molecular Immunology Unit, Department of Experimental Oncology, Fondazione IRCCS \\ "Istituto Nazionale dei Tumori", Milan, Italy \\ ${ }^{2}$ Department of Hematology, Oncology and Molecular Medicine, Istituto Superiore di Sanità, \\ Rome, Italy \\ ${ }^{3}$ Department of Human Pathology, University of Palermo, Palermo, Italy
}

OX40 stimulation is known to enhance activation of effector $\mathrm{T}$ cells and to inhibit induction and suppressive function of Treg. Here we uncovered a novel role of OX40 in sustaining Treg competitive fitness in vivo, during repopulation of lymphopenic hosts and reconstitution of BM chimeras. Defective expansion of OX40-null Treg diminished their ability to suppress inflammation in a model of lymphopenia-driven colitis. OX40-mediated promotion of Treg fitness spanned beyond lymphopenic environments, as endogenous Treg in OX40-null mice showed decreased accumulation during thymic development, enhanced susceptibility to antibody-mediated depletion and defective turnover following thymectomy. In vitro, OX40-deficient Treg were found to be intrinsically hyporesponsive to IL-2, in terms of Stat5 phosphorylation and proliferation, according to elevated SOCS1 content and reduced miR155 expression. Therefore, OX40 is a key factor in shaping Treg sensitivity to IL-2 and promoting their proliferation and survival, toward accurate immune regulation.

Key words: Competitive fitness - Costimulatory molecules - Cytokines - Treg

Supporting Information available online

\section{Introduction}

Treg are a specialized subset of $\mathrm{CD}^{+} \mathrm{T}$ lymphocytes deputed to maintain self-tolerance through inhibition of a variety of immune responses [1]. Dysregulation in functions or numbers of Treg has been associated to severe autoimmunity and inflammation in both mice and humans. Treg selectively express the transcription factor Foxp3, which determines their regulatory program, and constitutively express the $\alpha$-chain of the IL- 2 receptor CD25, which mediates their peripheral homeostasis.

Correspondence: Dr. Mario P. Colombo

e-mail: mario.colombo@istitutotumori.mi.it
Also, murine Treg constitutively express OX40 [2, 3], a member of the Tumor Necrosis Factor-Receptor superfamily, encoded by the Tnfrsf4 gene. OX40-null mice show defective T-cell activation and impaired reactivity in multiple settings [4]. Indeed, this receptor intervenes at different checkpoints in shaping optimal immune responses and in counteracting both recessive and dominant tolerance. OX40, expressed on non-Treg upon activation, conveys survival signals [5], promotes the acquisition of effector functions [6], impedes and reverts T-cell anergy [7] and even prevents the acquisition of a regulatory phenotype under TGF- $\beta$ exposure $[8,9]$ or under Tr1-promoting conditions [10]. A further level of control is represented by the

${ }^{*}$ These authors contributed equally to this work. 
ability of OX40 triggering to inhibit Treg suppressive function in vitro and in vivo, as shown in models of graft-versus-host disease [3], colitis [2] and cancer immunotherapy [11, 12]. Recently we have found OX40 bridging the bidirectional interaction between Treg and mast cells, leading to blockade of histamine degranulation and anaphylactic response on the mast cell side [13] and to inhibition of suppressive activity on the Treg side [14].

Little is known about the role of OX40 in Treg development and homeostasis. Young Tnfrsf $4^{-1-}$ mice show reduced numbers of $\mathrm{CD} 4{ }^{+} \mathrm{CD} 25^{+}$cells in central and peripheral lymphoid organs [2], though this observation has not been confirmed at the Foxp3 level. Lymphopenia-driven Treg proliferation is either decreased or increased in OX40L-null or OX40L-transgenic hosts, respectively, indicating a role for OX40 in enhancing Treg proliferation in vivo [2], but the functional relevance of this finding remains unexplored.

Crucial to Treg homeostasis is the IL-2 signal. In the absence of IL-2 or IL- 2 receptor, Treg still develop in the thymus but display a profound defect in peripheral homeostasis [15]. Treg developing from BM cells with impaired/absent IL-2 signal fail to compete in vivo for IL-2 during the reconstitution of lethally irradiated hosts $[16,17]$. The transcription factor Stat5, activated by IL-2, directly binds and induces Foxp3 [18]. On the other hand, Foxp3 sustains IL-2 responsiveness by inducing miR155, which inhibits at the post-transcriptional level SOCS1, a major silencer of IL-2 signal [19].

The signals of OX40 and IL-2 cooperate during the differentiation of conventional $\mathrm{T}$ cells into effector cells [20]; thus a similar interaction may also participate in Treg homeostasis. Indeed, we observed OX40 regulating the competitive fitness of Treg in vivo during repopulation of lymphopenic hosts, reconstitution of BM chimeras and suppression of inflammatory bowel disease. Endogenous Treg in Tnfrsf $4^{-/-}$mice show altered accumulation during thymic development, enhanced susceptibility to depletion and defective turnover following thymectomy, suggesting a role for OX40 in promoting Treg proliferation and survival in non-lymphopenic environments. OX40 deficiency is associated with a defective responsiveness to IL-2, in terms of STAT5 phosphorylation and cell proliferation. A higher SOCS1 level, due to a reduced miR155 expression, could account for impaired IL-2 response of OX40-null Treg. Therefore, OX40 sustains Treg fitness by lowering the threshold for IL-2 sensitiveness.

\section{Results}

\section{OX40 promotes Treg competitive fitness in vivo}

Lymphopenia-driven homeostatic proliferation is the suitable context in which differences in Treg competitive fitness may emerge. To study the direct competition between mature WT and Tnfrsf4 ${ }^{-/-}$Treg in vivo, Rag1 $1^{-/-}$mice received WT CD45.1 Treg mixed at 1:1 ratio with CD45.2 WT or Tnfrsf $4^{-1-}$ Treg (Fig. 1A). At day 20 after transfer, the CD45.1:CD45.2 proportion within circulating Foxp $3^{+}$cells was profoundly unbalanced in favor of WT Treg when cotransferred with OX40-null but not with WT counterparts (Fig. 1B). Time-course analysis of Treg frequency in peripheral blood showed that Tnfrsf $4^{-1-}$ Treg suffered the competition with WT Treg starting day 6 after transfer and progressively disappeared from the Treg compartment within the following $2 \mathrm{wk}$ (Fig. 1C and D, 18.98 \pm 0.95 versus $35.60 \pm 4.10$ at day $6, p<0.005 ; 6.18 \pm 1.09$ versus $32.88 \pm 3.40$ at day 13 , $p<0.005 ; 5.23 \pm 0.04$ versus $35.15 \pm 1.06$ at day $20, p<0.005$ ). The $\mathrm{CD}^{+}{ }^{+}$Foxp $^{-}$cells contaminating the preparation of adoptively transferred cells (around 10\%) extensively expanded in the lymphopenic hosts so as to recover the physiological Treg proportion, as previously reported [21]. We found no difference in the homeostatic expansion between WT and Tnfrsf $4^{-/-}$nonregulatory cells, indicating that OX40 signal enhanced competitive fitness selectively in Treg (Fig. 1D, right). The analysis of BrdU incorporation showed significantly lower division rate in Tnfrsf $4^{-1-}$ Treg compared with the WT control $(8.76 \pm 3.00$ versus $18.38 \pm 6.58, p<0.05)$, indicating that the defective competitiveness of Tnfrsf4 ${ }^{-1-}$ Treg was mainly due to decreased proliferation in response to lymphopenia (Fig. $1 \mathrm{E}$ and $\mathrm{F}$ ). The percentage of $\mathrm{Brdu}^{+}$cells in the gated CD45.1 Treg compartment did not differ when put in competition with WT or with Tnfrsf4 ${ }^{-/-}$CD45.2 Treg counterpart (Fig. 1E and F).

Two reports have shown that, following BM transplantation, host Treg surviving irradiation undergo extensive expansion, thus competing with donor-derived Treg, during the reconstitution of the normal Treg pool necessary to prevent lethal autoimmunity $[16,22]$. We analyzed the role of OX40 in the ability of developing donor Treg to compete with host residual cells by reconstituting lethally irradiated CD45.1 mice with T-cell-depleted BM cells obtained from scurfy mice, mixed at 4:1 ratio with WT or Tnfrsf $4^{-/-}$counterpart (Fig. 2A), using a previously described approach [23] that allows, in this combination, to obtain the totality of Treg from WT or Tnfrsf $4^{-/-}$BM while the majority of non-regulatory cells from scurfy (OX40-competent) BM. After 8-12 wk, peripheral blood, LN and spleens of all the chimeras showed a physiological Treg frequency (Fig. 2B). Based on the congenic marker CD45.1, the Treg pool contained a significant proportion of host radioresistant cells, which were OX40-positive, as expected (Fig. 2B). However, Treg derived from a Tnfrsf $4^{-1-}$ donor BM competed less efficiently with the OX40-expressing CD45.1 host Treg than Treg from WT donor BM. Indeed, the proportion of host:donor Treg shifted from 30:70 in scurfy: WT $>$ CD45.1 mice, to $50: 50$ in scurfy:Tnfrsf $4^{-/-}>$CD45.1 chimeras (Fig. 2B and C, $6.07 \pm 09 \%$ versus $8.72 \pm 0.96 \%$ donor Treg, $p<0.005 ; 5.51 \pm 0.63 \%$ versus $3.58 \pm 0.44 \%$ host Treg, $p<0.01)$. The OX40 effect on Treg competitive fitness was cellintrinsic, as no OX40-deficient cells other than Treg were underrepresented within the donor-derived compartment in scurfy:Tnfrsf4 $4^{-/-}>$CD45.1 chimeras. A kinetics experiment showed that BM-derived Tnfrsf $4^{-1-}$ Treg appeared in the peripheral blood at the same time of WT Treg, but showed defective competition to host Treg compared with the WT control starting $7 \mathrm{wk}$ after transplantation (Fig. 2D, left). Of note, the 
A

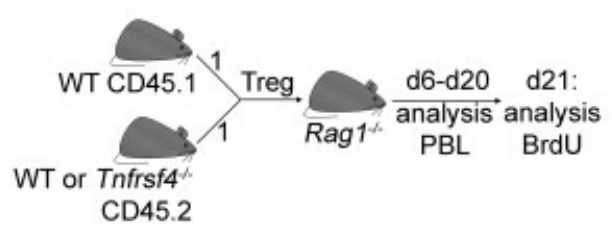

B

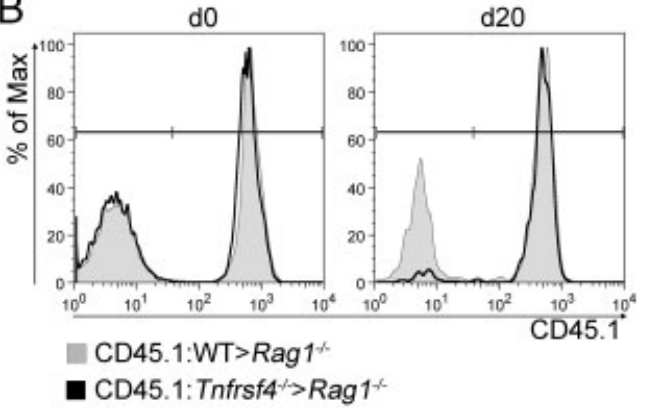

C
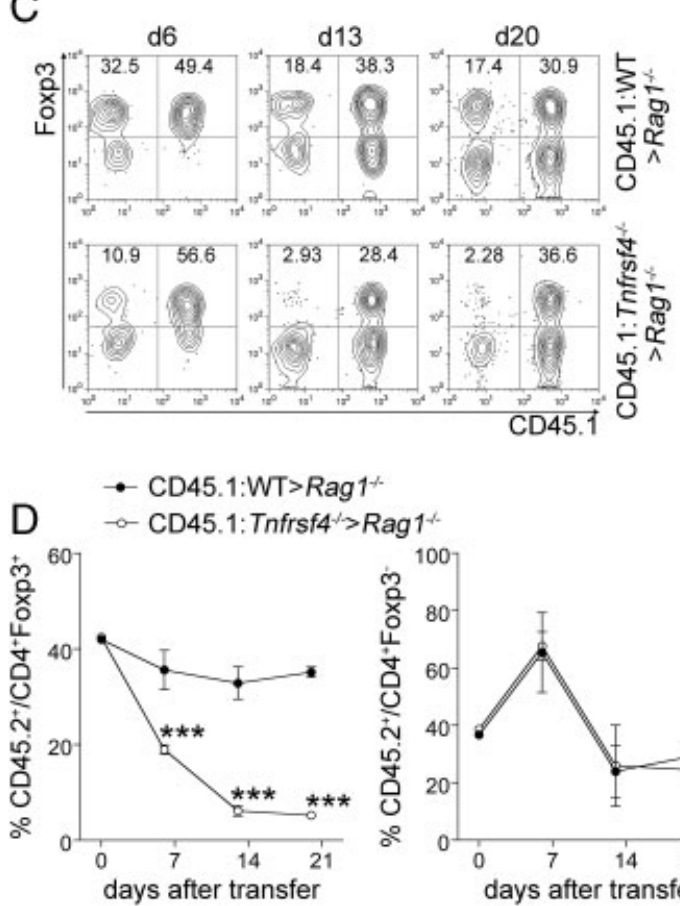

$$
\operatorname{Rag} 1^{*}
$$

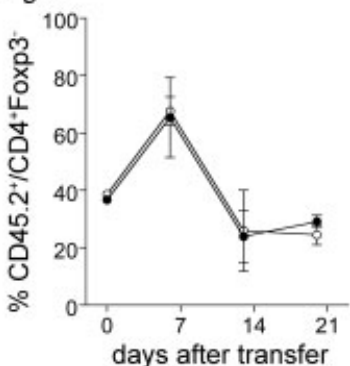

E

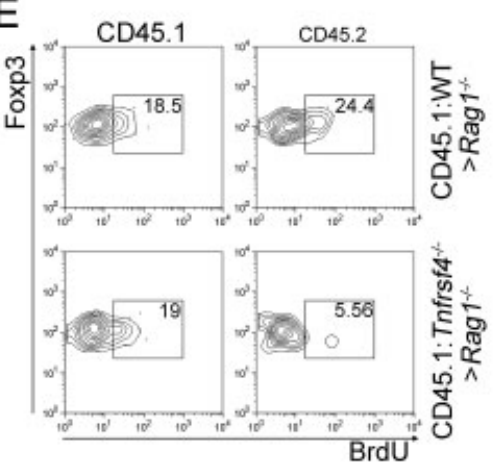

F

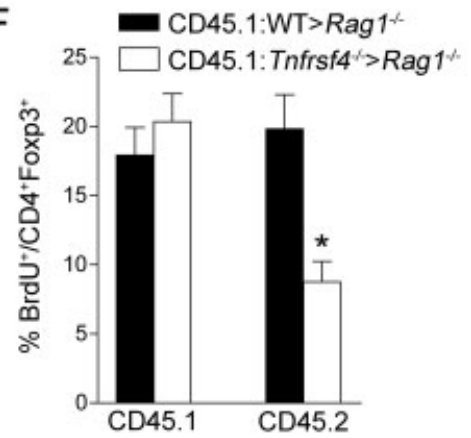

Figure 1. OX40 determines Treg competitive fitness in lymphopenia-induced proliferation. (A) Scheme of the experiment. WT CD45.1 Treg were mixed at 1:1 ratio with WTor Tnfrsf $4^{-/-}$CD 45.2 Treg and i.v. injected into Rag $1^{-1-}$ mice. Resultant cell populations were analysed by flow cytometry at the indicated times. (B) Representative histogram plots of CD45.1/CD45.2 frequency in gated CD $4^{+}$Foxp $3^{+}$Treg before injection and 20 days after in vivo expansion. (C) Representative contour plots showing Foxp3 versus CD45.1 staining of circulating Treg. (D) Frequency of CD45.2 cells in peripheral blood in the Treg (left) or non-Treg (right) compartments over time. (E) Representative plots of BrdU incorporation in CD45.1 or CD45.2 Treg in LN. (F) Pooled analysis of BrdU incorporation in CD45.1 or CD45.2 Treg in each experimental group. A mean 2.5-fold decrease in BrdU incorporation was detected in Tnfrfs $4^{-1-}$ as compared with WT Treg. Data are (B, C and E) representative or (D and F) mean \pm SD of four mice per group. The experiment was repeated twice. ${ }^{*} p<0.05,{ }^{* * *} p<0.005$ (non-paired Student's $t$ test).

Foxp3-negative compartment was replaced with BM-derived cells more efficiently than the Treg compartment and without difference between the two donor types, indicating again that OX40induced fitness was a Treg-restricted effect (Fig. 2D, right).

Thus, we have characterized the role of OX40 in sustaining Treg competitive fitness during homeostatic proliferation.

\section{Tnfrsf4 ${ }^{-1-}$ Treg show reduced capacity to inhibit lymphopenia-driven colitis}

Adoptively transferred $\mathrm{CD}^{+} \mathrm{CD}^{+} 5 \mathrm{RB}^{\text {high }} \mathrm{T}$ cells extensively proliferate into lymphopenic hosts, so as to induce a Th1- mediated inflammation mainly localized in the intestine and resembling inflammatory bowel disease [24]. Injection of Treg at the appearance of first signs of colitis results in symptoms amelioration and resolution of inflammation [25]. Exogenous Treg migrate to mesenteric LN, infiltrate colonic lamina propria, proliferate massively and suppress dendritic cells and $\mathrm{T}$ cells mainly through the local secretion of IL-10 [25, 26]. We took advantage of this experimental model to test whether the defective proliferation of Tnfrsf $4^{-/-}$Treg under lymphopenic condition was biologically relevant in the resolution of established colitis. $\mathrm{Rag}^{-/-}$mice repopulated with CD45.1 $\mathrm{CD}^{+}$ CD45RB ${ }^{\text {high }} \mathrm{T}$ cells, showing clinical signs of colitis such as weight loss, piloerection, hunching and diarrhea after 13 days, were left 
A

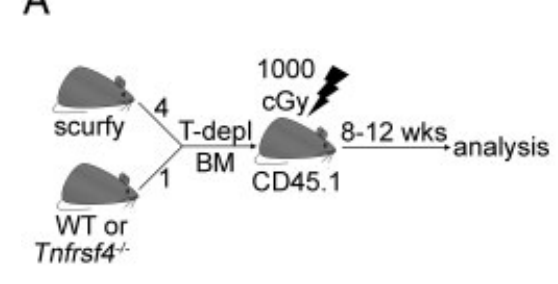

$\mathrm{B}$

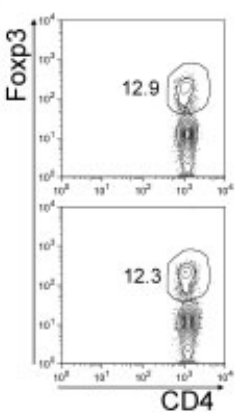

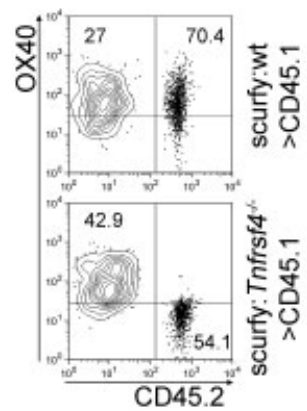
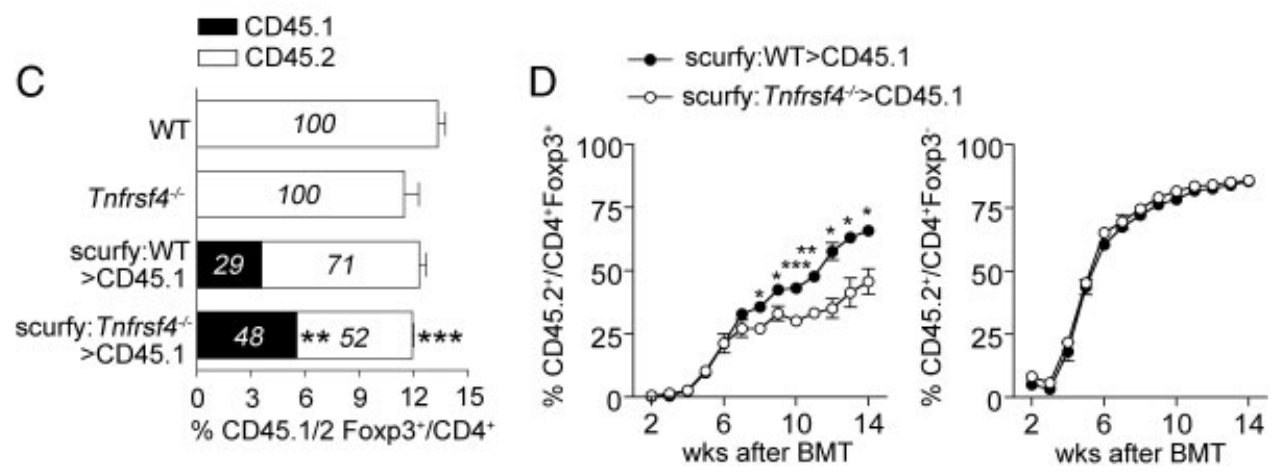

Figure 2. BM-derived OX40-null Treg compete less efficiently with host Treg in BM transplantation (A) Scheme of the experiment. CD45.1 mice were lethally irradiated and reconstituted with T-cell-depleted scurfy BM mixed in a 4:1 ratio with T-cell-depleted Tnfrsf $4^{-1-}$ or WT BM. (B) Representative flow cytometry of CD4 and Foxp3 staining (left panels) and the phenotype and frequency of host-derived (CD45.2-OX40 ${ }^{+}$) and BM-derived (CD45.2 $2^{+} \mathrm{OXO}^{+}$or $\mathrm{OX}_{40}^{-}$) cells in the gated Treg population (right panels) of LN cells from the two groups of BM chimeras. (C) Frequency and composition of the Treg pool in terms of CD45.1/CD45.2 subsets in BM chimeras as compared with WT and Tnfrsf4 ${ }^{-1-}$ controls. Numbers indicate the mean percentage of CD45.1 or CD45.2 cells in each group. (D) Kinetics of cell repopulation in the peripheral blood of CD45.2 BM-derived Treg (left) and non-Treg (right). Data are (B) representative and (C-D) mean \pm SD of three to five mice per group. The experiment was repeated five times. ${ }^{*} p<0.05,{ }^{* *} p<0.01,{ }^{* * *} p<0.005$ (non-paired Student's $t$ test).

untreated or received CD45.2 WT or Tnfrsf $4^{-1-}$ Treg. While untreated mice failed to resolve the disease, mice injected with WT Treg showed clinical improvement and increased body weight. On the contrary, Tnfrsf4 ${ }^{-1-}$ Treg showed a significantly lower ability to cure colitis, as recovery of the initial body weight occurred with a significant delay (from d23-d31 to d32-d41 interval) compared with WT Treg-cured controls (Fig. 3A). On histopathological evaluation untreated mice displayed a severe colitis (median score 4, mean score $3.50 \pm 0.71$ ) with marked disruption of the glandular epithelium, profound reduction of the goblet cell number and intense inflammatory infiltration involving both mucosa and submucosa. Treatment with WT Treg achieved histological remission of the colitis (median score 1 , mean score $1.40 \pm 0.47, \quad p<0.005$ versus untreated $p<0.05$ compared with Tnfrsf $4^{-1-}$ Treg) since treated mice showed only slight crypt hyperplasia, scant leukocytic infiltration and normal to slightly reduced number of goblet cells. Specimens of mice treated with Tnfrsf4 ${ }^{-1-}$ Treg were characterized by moderate hyperplasia of the crypts, reduced number of goblet cells and considerable chronic inflammatory infiltrate mainly involving the lamina propria (median score 2, mean score $1.93 \pm 0.78, p<0.005$ ), consistently with an intermediate picture between untreated and WT Treg-treated mice. Serum TNF- $\alpha$, measured in the $\mathrm{d} 32-\mathrm{d} 41$ interval, was significantly reduced in colitic mice, receiving WT but not Tnfrsf $4^{-/-}$
Treg (175.84 \pm 103.49 in untreated mice, versus $59.69 \pm 38.71$ with WT Treg, $p<0.05$; versus $98.99 \pm 76.06$ with Tnfrsf $^{-1-}$ Treg, $p>0.05$ (Fig. 3B and C).

To correlate disease manifestation with Treg expansion, we checked the frequency of Treg in peripheral blood at two different time points during the resolution of colitis. As previously reported, we found a sizeable population of Foxp $3^{+}$cells carrying the CD45.1 congenic marker, derived from the conversion into Treg of non-regulatory precursors contained in the donor $\mathrm{CD}^{+}$ CD45RB ${ }^{\text {high }} \mathrm{T}$ cells [27]. Twenty-three days after colitis induction, when mice treated with WT Treg showed accelerated weight gain compared with untreated and Tnfrsf $4^{-1-}$ Treg-treated controls, we found a higher frequency of Foxp $3^{+}$cells in mice injected with WT Treg than in untreated mice, the majority of which comprised CD45.2 exogenous Treg (Fig. 3). On the contrary, the fraction of Treg in mice injected with Tnfrsf4 ${ }^{-1-}$ Treg was similar to that of untreated controls and contained a majority of CD45.1 induced Treg (Fig. 3D, 57.66 $\pm 16.81 \%$ CD45.2 Treg in the group treated with WT cells versus $37.10 \pm 6.83 \%$ in the group treated with Tnfrsf4 ${ }^{-/}$Treg, $p<0.05)$. In contrast, at day 34 , when mice treated with Tnfrsf $4^{-/-}$Treg eventually started to ameliorate, CD45.2 Treg expanded and outnumbered the CD45.1 counterpart without difference with mice injected with WT Treg (Fig. 3D). High Treg frequency was detected also in the mesenteric LN and in the 
A

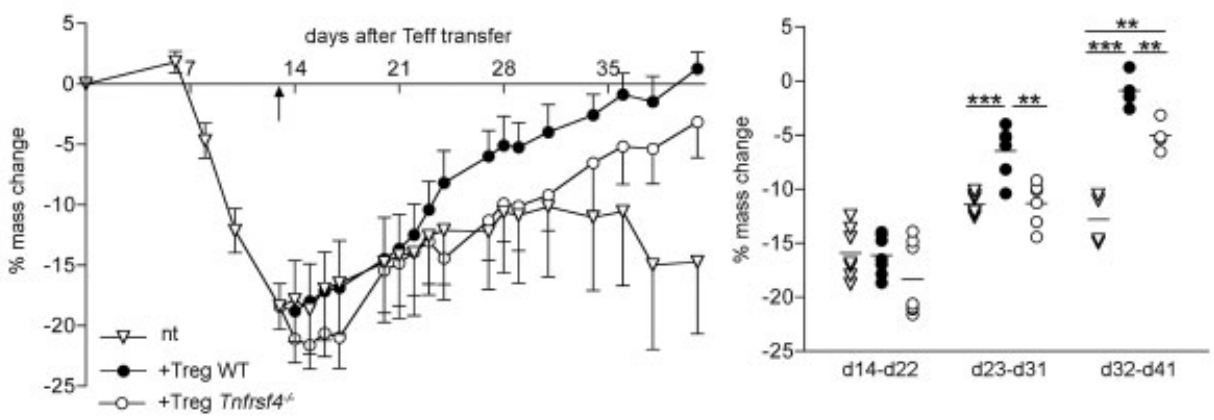

B

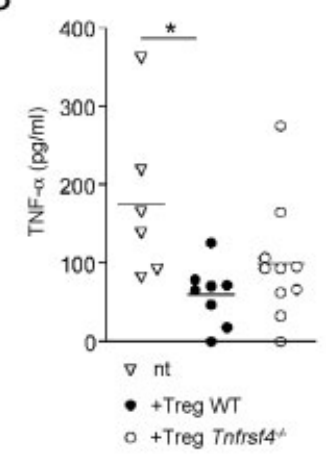

D

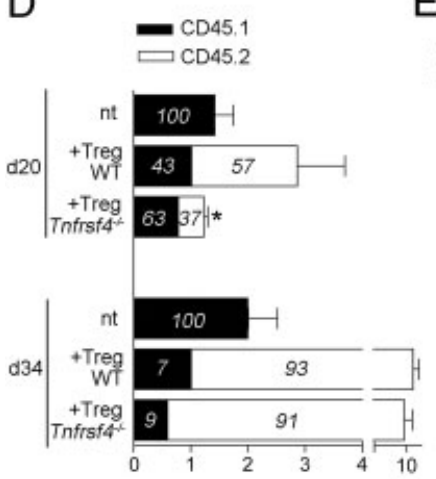

C
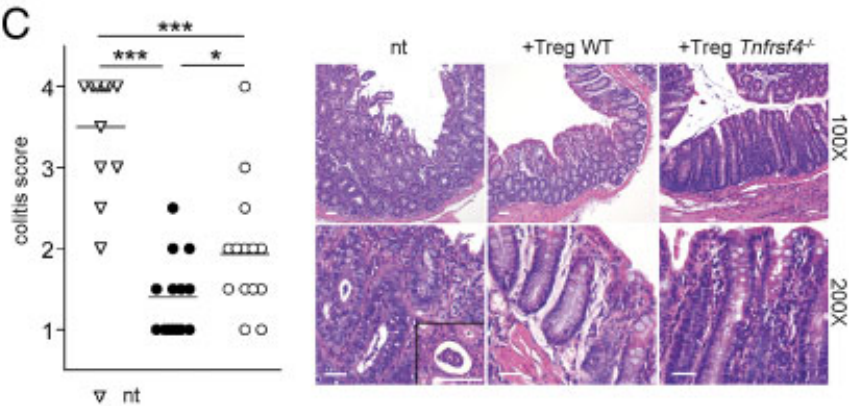

- +Treg WT

$0+$ Treg Tnfrsf $4^{4}$

E

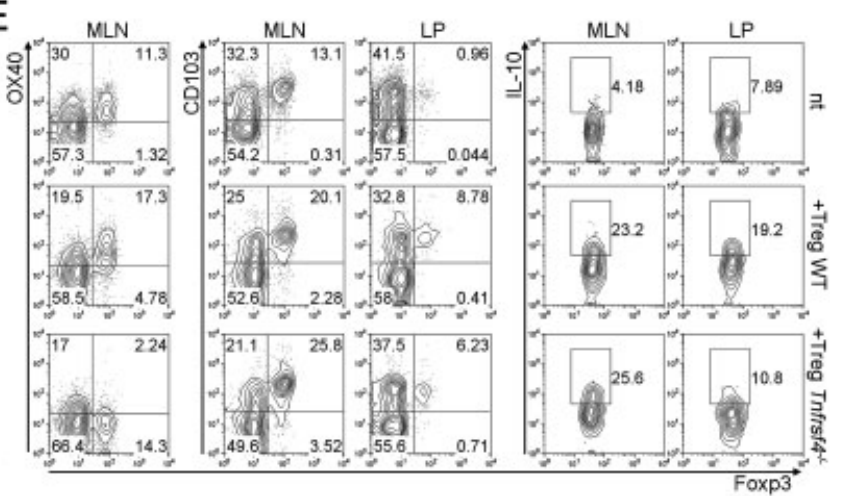

Figure 3. OX40-null Treg have decreased ability to resolve colitis. Colitis was induced in Rag $1^{-/-}$mice by injecting sorted CD45.1 CD45RB high $C D 4^{+}$ cells (day 0). At day 13 (arrow), mice were left untreated (nt) or received WT or Tnfrsf $4^{-1-}$ Treg. (A) Kinetics of percent change in body weight (left; mean \pm SD) and percent change in body weight grouped by time intervals (right; each symbol represents one mouse; short horizontal bar, the mean). (B) Serum TNF- $\alpha$ levels on day 37 after colitis induction. (C) Histological grading of colitis on day 42. Colitis score per animal (left; horizontal bar represents the mean) and representative H\&E-stained sections from the distal portion of the colon (right). The inset shows cryptic abscesses. Scale bars (white): $50 \mu \mathrm{m}$. Original magnifications: upper panels, $100 \times$; lower panels $200 \times$; inset $400 \times$. (D) Frequency and proportions of circulating CD45.1/CD45.2 Treg on day 20 or 34. Numbers indicate the mean percentage of CD45.1 or CD45.2 cells in each group. (E) Expression of Foxp3 versus OX40, CD103 or IL-10 by mesenteric LN (MLN) and lamina propria (LP) cells. The experiment was repeated twice with four to five mice per group. ${ }^{*} p<0.05,{ }^{* *} p<0.01,{ }^{* * *} p<0.005$ (non-paired Student's $t$ test).

lamina propria of mice treated with WT or OX40-null Treg, in comparison to untreated mice (Fig. 3E). This suggests that OX40 plays a non-redundant role in early Treg expansion and colitis suppression, and that other signals may overcome OX40 deficiency and rescue Treg fitness and function at later time points.

Beside defective systemic expansion, impaired suppressive function and/or suboptimal recruitment of OX40-null Treg might concur to inefficient inhibition of colitis. Because of the established role of Treg-derived IL-10 in suppressing colitis [26], we tested whether Tnfrsf $4^{-/-}$Treg could have defective suppressive function in vivo as a consequence of reduced IL-10 production. IL-10 intracellular staining performed in Treg re-populating mesenteric $\mathrm{LN}$ and colonic lamina propria showed no relevant defect in IL-10 secretion in WT or Tnfrsf $4^{-/-}$ Treg (Fig. 3E). To also investigate the possibility of a defective recruitment of Tnfrsf $4^{-/-}$Treg to inflamed sites, Treg were stained for $\mathrm{CD} 103$, a crucial integrin for gut homing and for immune regulation in the colon $[28,29]$. Both WT and OX40-null Treg were found highly expressing CD103 without difference (Fig. 3E). 


\section{OX40 governs Treg fitness in non-lymphopenic contexts}

BM transplantation and T-cell transfer in immunodeficient hosts are characterized by extensive lymphopenia-driven proliferation, a setting that although non-physiological may mirror some therapeutic situations. Nevertheless, to address the contribution of OX40 in shaping the endogenous Treg compartment in immunocompetent mice, we analyzed the frequency of Foxp $3^{+}$ $\mathrm{T}$ cells in peripheral lymphoid organs and thymuses of WT and Tnfrsf $4^{-1-}$ mice at different ages. While no difference was found in Treg frequency in LN (Fig. 4A and B) or spleens (data not shown), CD4 single positive, but not double positive, thymocytes of OX40-null animals contained a significantly lower percentage of $\mathrm{Foxp}^{+}$cells regardless of mouse age (Fig. 4A and B, $3.46 \pm 0.41$ versus $4.77 \pm 0.44$ at $2 \mathrm{wk}, p<0.01 ; 3.03 \pm 0.50$ versus $3.98 \pm 0.55$ at $4 \mathrm{wk}, p<0.05 ; 2.25 \pm 0.36$ versus $3.40 \pm 0.25$ at $8 \mathrm{wk}, p<0.01)$. We did not detect an altered expression level of Foxp3 or CD25 in thymic or peripheral Treg in WT or Tnfrsf $4^{-/-}$ mice at any age (data not shown). These results indicate that OX40 may play a physiological role in Treg thymic expansion, rather than in Treg lineage decision at the double positive stage.

The fitness signal triggered by OX40 in Treg may extend beyond proliferation and directly affect their survival under external pressures, for instance mediating their resistance to depletion mediated by anti-CD25 mAb (PC61). Indeed, we observed that Treg resisting PC61-mediated depletion displayed higher expression of OX40 6 days after treatment than untreated Treg (Supporting Information Fig. 1A and B, 127.50 \pm 11.61 versus $82.25 \pm 7.05, p<0.005)$. Accordingly, Tnfrsf $4^{-/-}$Treg were significantly more susceptible to depletion than WT Treg (Supporting Information Fig. $1 \mathrm{C}$ and $\mathrm{D}, 4.95 \pm 0.70$ versus $6.64 \pm 0.83 \%$ residual Treg, $p<0.005)$. We also checked the response of WT and Tnfrsf $4^{-1-}$ Treg to central depletion operated by thymectomy. Three weeks after thymus removal, Treg frequency was significantly lower in $T n f r s f 4^{-/-}$than WT mice $(14.15 \pm 2.30$ versus $16.72 \pm 2.18, p<0.05)$. The Treg decline in Tnfrsf $^{-/-}$versus WT mice was associated to significant increase

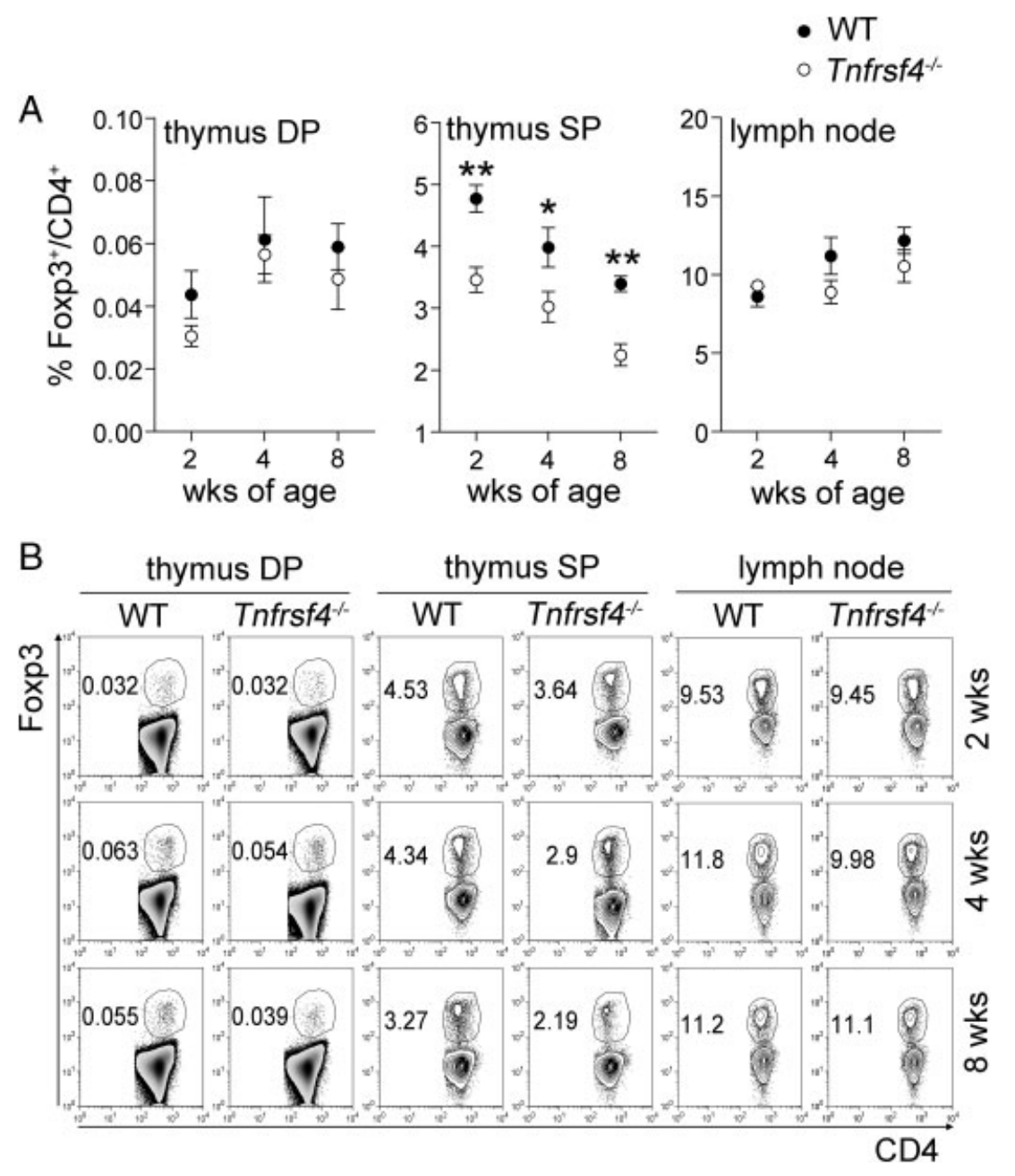

Figure 4. Role of OX40 in Treg thymic development. Treg frequency in thymuses and peripheral LN of WT and Tnfsrf $4^{-1-}$ mice aged 2, 4 or 8 wk. (A) Mean \pm SD of four mice per group and (B) representative plots of CD4 and Foxp3 staining at the indicated ages. DP, double positive thymocytes; SP, CD4-single positive thymocytes. ${ }^{*} p<0.05,{ }^{* *} p<0.01$ (non-paired Student's $t$ test). 
of apoptosis $\left(4.16 \pm 3.03\right.$ versus $1.64 \pm 0.89 \%$ AnnexinV $^{+}$Treg, $p<0.05)$ and decrease of proliferation $(5.38 \pm 1.56$ versus $7.56 \pm 2.06 \% \mathrm{BrdU}^{+}$Treg, $p<0.01$ ), indicating a role for OX40 in supporting Treg turnover in this context (Supporting Information Fig. 2). In summary, OX40 supported the fitness of endogenous Treg also in non-lymphopenic hosts, especially when Treg should maximize the response to trophic factors, such as during thymic development and depletion-induced peripheral expansion.

\section{OX40 signal optimizes Treg responsiveness to IL-2}

IL-2 is the main cytokine responsible for Treg fitness in vitro and in vivo [30]. We hypothesized that the lower ability of Tnfrsf $4^{-1-}$ Treg to expand in lymphopenic conditions might result from a defective response to IL-2. WT and Tnfrsf4 ${ }^{-1-}$ Treg were stimulated in vitro with scaled doses of recombinant IL-2 and stained for phosphorylated STAT5 after different time intervals. STAT5 phosphorylation was lower in Tnfrsf $4^{-/-}$than in WT Treg, following exposure to different concentrations of IL-2 (Fig. 5A). This event was more pronounced after 5 or $10 \mathrm{~min}$ than after
1 min, suggesting that the defect of OX40-null Treg mainly affected late and sustained, rather than immediate, IL-2 response (Fig. 5B). To study whether impaired IL-2 response was correlated to reduced proliferation, we exposed CFSE-labelled WT and Tnfrsf $4^{-1-}$ Treg to scaled concentrations of IL-2 in the presence of TCR triggering. Without exogenous IL-2, Treg were completely anergic, as previously described [31]. When IL-2 was present, OX40-deficient Treg proliferated with lower efficiency compared with their WT counterparts (Fig. 5C and D), in line with the attenuated STAT5 phosphorylation. We checked the expression level of CD25 in WT and Tnfrsf $4^{-/-}$Treg, freshly isolated ex vivo or after in vivo expansion, without finding any difference (data not shown). Therefore, we hypothesized that attenuated IL-2 responsiveness in OX40-null Treg might be due to an intrinsic defect in the intracellular machinery leading to STAT5 signaling.

SOCS1 is one of the major inhibitors of STAT5 phosphorylation following engagement of IL-2 receptor [32]. Rudensky and colleagues have recently demonstrated that increased expression of SOCS1 is responsible for the diminished competitive fitness of miR155 ${ }^{-1-}$ Treg [19]. Indeed, Foxp3 directly induces miR155 that in turn silences SOCS1 expression, thus enhancing IL-2

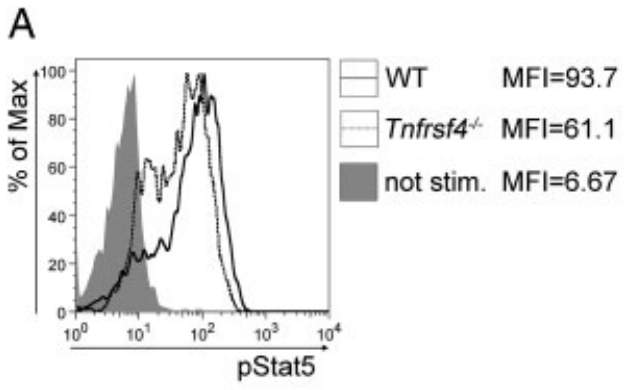

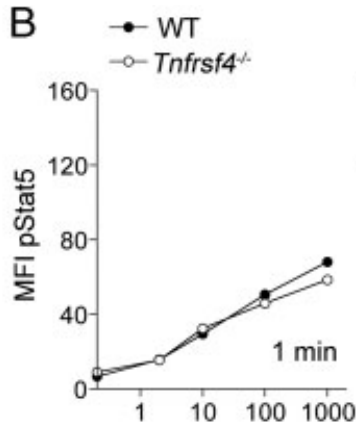

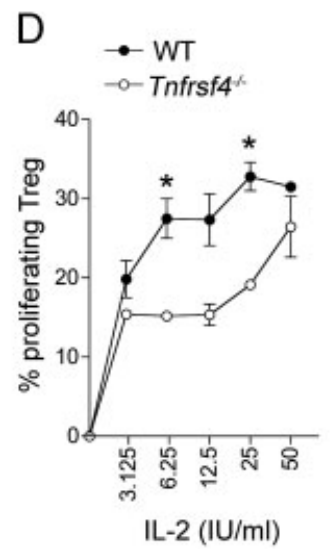

E
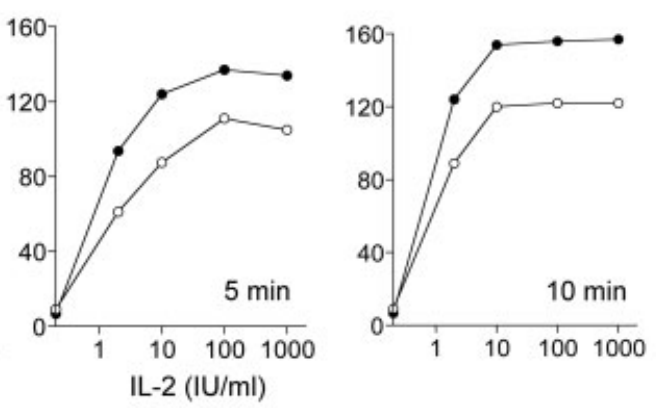

$\mathrm{F}$

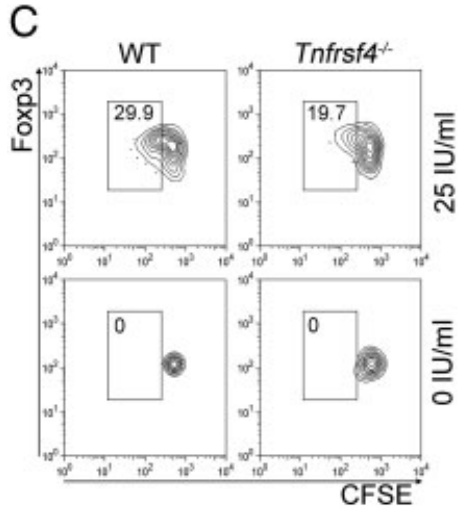

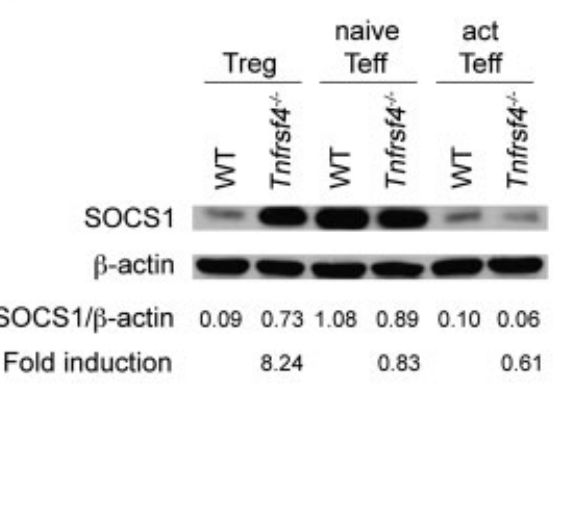

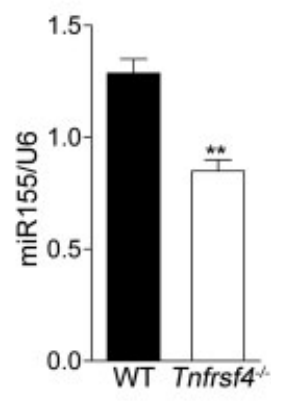

Figure 5. Attenuated IL-2 signaling in OX40-deficient Treg. (A and B) Purified WT or Tnfrsf4 ${ }^{-1-}$ Treg were left unstimulated or treated with increasing doses of rIL-2 for the indicated times and phospho-Stat5 determined by flow cytometry. (A) Representative histograms (at 5 min with $2 \mathrm{IU} / \mathrm{mL}$ rIL-2) and (B) kinetics of Stat5 phosphorylation. (C and D) In vitro proliferation of WT or Tnfrsf4 ${ }^{-1-}$ Treg after $72 \mathrm{~h}$ exposure to anti-CD3 and increasing concentrations of rIL-2. (C) Representative plots of CFSE dilution at $72 \mathrm{~h}$ in gated $\mathrm{CD} 4^{+}$Foxp $3^{+}$Treg and (D) the proliferative response of Treg to increasing doses of IL-2 doses. (E) Western blot of SOCS1 in purified Treg or non-regulatory cells (Teff), naive or activated for 3 days with anti-CD3, obtained from WT or Tnfrsf $4^{--}$mice. Numbers indicate densitometric SOCS1 expression values normalized to $\beta$-actin expression values and fold increase in SOCS1 expression in OX40-null cells compared with WT cells. (F) Quantitative Real-time RT-PCR of miR155, normalized to U6, in WT or Tnfrsf $4^{-1-}$ Treg. Every experiment was repeated twice with pooled Treg from five to ten mice per group. (A, B, C and E) Representative and (D and F) mean \pm SD data of two experiments. ${ }^{*} p<0.05,{ }^{* *} p<0.01$ (non-paired Student's $t$ test). 
signal [19]. To test whether the diminished fitness of Tnfrsf $4^{-1-}$ Treg involved dysregulation in this pathway, we checked SOCS1 protein level in WT and Tnfrsf4 $4^{-/-}$Treg by Western blot. As controls, naïve and activated $\mathrm{T}$ cells were used, expected to express high and low level of SOCS1, respectively. We found Tnfrsf $4^{-/-}$Treg expressing an eight-fold higher SOCS1 level than the WT counterpart, while no relevant differences were observed in naïve or activated T cells (Fig. 5E). In line with this result, real time RT-PCR revealed diminished miR155 amounts in Tnfrsf4 ${ }^{-/-}$ compared with WT Treg $(0.85 \pm 0.10$ versus $1.29 \pm 0.13, p<0.01)$, indicating that miR155 dysregulation may lie behind altered SOCS1 expression and IL-2 response (Fig. 5F). These data indicate that OX40-null Treg have an intrinsic defect in IL-2 responsiveness because of decreased miR155 content and consequently enhanced SOCS1 expression.

\section{Discussion}

OX40 has emerged as a relevant molecule regulating Treg suppressive functions. Several findings suggest that OX40 role in Treg biology may reveal a higher degree of complexity than previously expected [33]. Here we show that OX40 expression has a non-redundant role in supporting Treg competitive fitness in vivo in both lymphopenic and lymphoreplete environments. The underpinning mechanism stems from a constitutive lower sensitivity to IL-2, due to decreased miR155 content and consequently increased SOCS1 expression.

Impaired competitive fitness of Tnfrsf $4^{-/-}$Treg resulted in their lower efficiency to cure lymphopenia-driven colitis. Indeed, in this model, extensive Treg proliferation occurs in vivo so as to outnumber effector cells and resolve inflammation [25]. However, we cannot exclude that OX40 deficiency may compromise also the intrinsic suppressive function of Treg. For instance, we have shown that Treg suppress histamine degranulation in mast cells through OX40 [13], and Khazaie and colleagues have demonstrated that adoptively transferred Treg inhibit mastocytosis in colon polyposis [34], but very little is known about involvement of this pathway in colitis development and resolution. We have checked for IL-10 production, a critical factor in colitis suppression [26, 35, 36], among lamina propria-infiltrating Treg, and found no difference between OX40-proficient and OX40-deficient Treg. Although we have previously shown a similar surface phenotype in naïve WT and OX40-null Treg [13], we cannot exclude that other molecules, such as Pd-1, CTLA-4 and membrane-bound TGF- $\beta$, are differentially expressed in Tnfrsf4 ${ }^{-1-}$ Treg from inflamed tissues where they may become relevant for Treg-intrinsic suppressive ability. However, expression of CTLA-4 or Pd-1 may not be exclusively interpreted as function of Treg suppressive activity, being also associated to Treg maintenance and expansion [37, 38]. However, defective Treg recruitment into inflamed organs seems unlikely, WT and Tnfrsf $4^{-/-}$Treg being present in comparable frequency and with similar CD103 expression pattern in mesenteric LN and lamina propria of cured mice.
When transferred at 1:1 ratio into $\operatorname{Rag} 1^{-/-}$mice, CD45.2 Tnfrsf $4^{-/-}$Treg were greatly outnumbered by the CD45.1 WT counterpart and almost disappeared from lymphoid organs (Fig. 1) including mesenteric $\mathrm{LN}$ and colonic lamina propria (data not shown). Conversely, in $\mathrm{Rag}^{-/-}$mice receiving CD45.1 $\mathrm{CD}^{+} \mathrm{CD}^{+} 5 \mathrm{RB}^{\text {high }}$ to induce colitis, the minority of CD45.1induced WT Treg initially dominate the competition with the Tnfrsf $4^{-/-}$Treg given for therapeutic purpose, but lately the Tnfrsf $4^{-1-}$ Treg expanded and outcompeted the CD45.1 fraction. The different outcome could be due to the starting proportion and the nature of competitors that may shift the balance of competition. Indeed OX40-null Treg compete with equal amounts of natural Treg in the former setting, while with a minority of induced Treg in the latter setting. Importantly, natural and induced Treg display a genetically different program that may affect their phenotypic stability in vivo [27].

Many data support the idea that OX40/OX40L drives inflammation in different models of experimental colitis. Dendritic cells highly express OX40L in the mesenteric LN of colitic mice [39]. Blockade of this axis by specific antibodies dramatically reduces T-cell activation and development of colitis, induced by T-cell transfer [39, 40] or dextran sulfate sodium [41]. Tnfrsf $4^{-/-}$ $\mathrm{T}$ cells are not able to induce colitis into $\mathrm{Rag} 2^{-/-}$mice, and WT $\mathrm{T}$ cells failed to induce colitis into OX40L-deficient $\mathrm{Rag} 2^{-/-}$mice, observations that demonstrate that OX40 triggering is crucially required for activation of colitogenic $\mathrm{T}$ cells [2]. On the other hand, OX40 triggering inhibits Treg suppression, as demonstrated by the finding that Treg cannot suppress colitis when cotransferred with $\mathrm{T}$ cells in transgenic immunodeficient mice expressing OX40L ubiquitously and constitutively [2]. We add here that Tnfrsf $4^{-1-}$ Treg show diminished ability to cure established colitis, because of an intrinsically defective lymphopenia-driven proliferative response.

A previous report highlighted that young OX40-deficient mice have lower counts of $\mathrm{CD} 4{ }^{+} \mathrm{CD} 25^{+} \mathrm{T}$ cells in both spleen and thymus [2]. However, a more recent study with Foxp3gfp knock-in mice did not highlight any major defect in Treg frequency in lymphoid organs of OX40-KO mice [9]. According to the latter, we found no difference in Treg frequency or Foxp3 content in spleens or LN of WT and Tnfrsf4 ${ }^{-1-}$ mice. Refined analysis of thymocytes revealed decreased Treg percentage in CD4 single positive, but not double positive, $\mathrm{T}$ cells in OX40-null mice. Indeed, OX40 is expressed by developing thymocytes, particularly by Treg precursors, mainly at the single positive stage [42]. OX40 induction in Foxp $3^{+}$thymocytes accompanies high TCR expression and may even precede CD25 up-regulation [42]. Of note, the amount of miR155 is largely expanded in single positive compared with Foxp $3^{+}$double positive thymocytes [19], indicating that optimal IL-2 responsiveness, settled by OX40, CD25 and miR155 expression, intervenes in shaping Treg thymic pool without compromising early Treg development. This defect is however compensated in the periphery, likely because regulatory thymic emigrants rapidly accumulate under peripheral homeostatic control. 
Treg surviving depletion regimens based on polyclonal antilymphocyte serum [43] or cyclophosphamide [12] express higher levels of OX40 than the bulk untreated Treg population. We have confirmed this finding in mice treated with the anti-CD25 antibody PC61, widely used to deplete Treg. PC61 fails to completely eliminate Treg and down-regulates the expression of CD25 in residual Treg [44]. According to previous findings regarding depletion by polyclonal anti-lymphocyte serum [43], we found OX40-null Treg more susceptible to PC61-induced depletion. We could not formally distinguish whether Treg already expressing high levels of OX40 were protected from depletion, whether Treg surviving depletion up-regulate OX40 to maximize their fitness in vivo or whether OX40-competent Treg proliferate more efficiently in response to depletion. In thymectomized animals, both increased apoptosis and decreased proliferation concurred to the Treg decline observed in Tnfrsf $4^{-/-}$mice, suggesting that defective Treg response to fitness signals may, directly or indirectly, compromise several aspects of their biological functions. OX40 appears an important signal in sustaining Treg expansion/survival, especially in condition of suboptimal sensitiveness to IL-2 because of down-regulation of the CD25 molecule. However, the sole OX40 deficiency was not sufficient to allow complete Treg depletion following PC61 treatment, suggesting that other signals may contribute to Treg survival to depletion.

OX40 triggering has been interpreted so far as a counterregulatory mechanism, as it prevents TGF- $\beta$-induced Treg development and counteracts Treg suppressive function. We have found here that OX40-null Treg show attenuated fitness and decreased colitis suppression, attributing to OX40 a key role in promoting immune regulation. Apparently, the same signal that inhibits Treg function sustains their fitness in vivo. An explanation may be that Tnfrsf $4^{-1-}$ Treg have intrinsic defects in their fitness, possibly due to an altered transcriptional program established during thymic development. Indeed, we found Tnfrsf $4^{-1-}$ Treg intrinsically hyporesponsive to IL-2 in vitro, in the absence of an OX40 triggering signal. Therefore, pharmacological or genetic OX40 triggering on mature WT Treg may inhibit their suppressive function, while genetic OX40-deficiency may lead to impaired Treg fitness. The cytokine context in which OX40 engagement occurs may dictate the outcome of stimulation. Indeed, it could be proposed that in homeostatic conditions dominated by IL-2, such as thymic development and lymphopenia-induced proliferation, OX40 enhances Treg susceptibility to IL-2 thus promoting Treg fitness; conversely, in inflammatory conditions dominated by proinflammatory cytokines, OX40 may expose Treg to microenvironmental cues subverting their regulatory program. This hypothesis requires further investigation, especially in relation to Foxp3 expression level in Treg following OX40 engagement: we could not find any difference in Foxp3 protein amount in WT or Tnfrsf $4^{-/-}$Treg, while others have observed decreased Foxp3 content at both mRNA and protein level in OX40-triggered Treg [9].

The context of OX40 engagement could also dictate whether intratumoral Treg will be simply inhibited or even eliminated by an OX40 agonist. We have showed that tumor-infiltrating Treg express higher levels of OX40 compared with systemic Treg and that intratumoral injection of an OX40 agonist induced tumor rejection through the inhibition of Treg suppression [14]. Conversely, others have shown that, following cyclophosphamide-based Treg depletion, OX40 is further up-regulated on survived Treg and locally conveys death signals as a result of hyper-activation [12]. It could be speculated that OX40 effects on Treg may span from fitness to inhibition to death in different contexts.

IL-2 regulates the intratumoral balance between Treg and Th17 cells, favouring the former and counterbalancing the latter [45]. Stat5 phosphorylation may dictate the balance between the two lineages, constraining Th17 skewing [46] while supporting Foxp3 expression [18]. OX40 may thus play a role in the Th17-Treg balance in pathological conditions such as cancer and autoimmunity. In a mouse model of CNS-directed autoimmunity, OX40 expression can be detected on Th17 cells [14].

We have recently shown that Treg directly suppress mast cell degranulation through OX40 [13]. This observation further supports the idea that this molecule may play different roles in Treg suppressive function, depending on the kind of target cells, the strength of stimulation, the cytokine microenvironment, the stability of Foxp3-induced program and the expression of other costimulatory molecules [33]. Intrinsic to the costimulatory nature of OX40 is the fact that, in all the aforementioned contexts, its engagement does not provide an on/off switching effect, but rather a fine-tuning of the cellular response to external challenges.

\section{Materials and methods}

\section{Mice and treatments}

C57BL/6 WT mice were purchased from Charles River (Italy). C57BL/6 CD45.1-congenic mice and $\mathrm{Rag}^{-/-}$mice were from Jackson Laboratories. C57BL/6 OX40-deficient mice (Tnfrsf4 ${ }^{-{ }^{-}}$) were from Nigel Killeen (UCSF). Scurfy mice were from Anna Villa (HSR-TIGET, Milan, Italy). Mice were maintained under pathogen-free conditions at the animal facility of Fondazione IRCCS "Istituto Nazionale dei Tumori". Animal experiments were authorized by the Institute Ethical Committee and performed in accordance with institutional guidelines and national law (DL116/92). In vivo systemic administration of anti-CD25 mAb (PC61; hybridoma provided by V. Bronte, Padua, Italy) was performed as previously described $[11,13]$.

\section{Treg purification and adoptive transfer}

Total splenocytes were enriched for $\mathrm{T}$ lymphocytes by passing cells on nylon wool columns (Kisker). $\mathrm{CD}^{+}$and $\mathrm{B}^{+} 2 \mathrm{O}^{+}$cells were removed using anti-CD8 and anti-B220 microbeads 
(Miltenyi Biotec). $\mathrm{CD} 4^{+} \mathrm{CD} 25^{+}$cells were then separated using the $\mathrm{CD} 25^{+}$T-cell isolation kit (Miltenyi Biotec), according to the manufacturer's instructions. Flow cytometry showed that the separate fractions were $90 \%$ pure from both WT and Tnfrsf $4^{-/-}$ mice. A total of $4 \times 10^{5} \mathrm{CD} 45.1 \mathrm{WT}$ Treg were mixed with equal amount of CD45.2 WT or Tnfrsf4 ${ }^{-/-}$Treg and i.v. injected into Rag $1^{-/-}$mice.

\section{BM transplantation}

BM cells were obtained by flushing the cavity of femurs from donor mice. T-cell depletion was performed by magnetic separation with anti-CD5 microbeads (Miltenyi Biotec). Cells from scurfy male mice were mixed at 4:1 ratio with CD45.2 WT or Tnfrsf4 ${ }^{-1-}$ cells. CD45.1 B6-recipient male mice were lethally $\gamma$ irradiated with $1000 \mathrm{cGy}$ (given as a split dose $500+500$ cGy with a 3-h interval). After $2 \mathrm{~h}$, mice were injected i.v. with $6-9 \times 10^{6}$ mixed $\mathrm{BM}$ cells. Recipient mice received $0.4 \mathrm{mg} / \mathrm{mL}$ gentalyn in the drinking water starting $1 \mathrm{wk}$ before irradiation and maintained thereafter.

\section{In vivo BrdU incorporation and staining}

Mice were injected i.p. once with $1 \mathrm{mg}$ of BrdU (Sigma-Aldrich) and then given $0.8 \mathrm{mg} / \mathrm{mL}$ BrdU in drinking water for 3 days. LN and spleen cells were surface stained, fixed with Fix/Perm buffer (eBioscience) and then analyzed using the BrdU Flow kit (BD Pharmingen). Antibodies to BrDU (BD Pharmingen) and Foxp3 (eBioscience) were added simultaneously to permeabilized cells.

\section{Induction, cure and analysis of colitis}

T cells, obtained from CD45.1 WT mice by passing spleens on nylon wool columns (Kisker), were enriched for $\mathrm{CD}^{+}$by negative selection through magnetic separation with anti-CD8 and anti-B220 microbeads (Miltenyi Biotec). Cells were stained with FITC anti-CD45RB (16A, eBioscience) and CD45RB ${ }^{\text {high }}$ cells were sorted on a FACSAria (Becton Dickinson). Purity was $>99 \%$. A total of $5 \times 10^{5}$ sorted cells were i.p. injected into Rag $1^{-/-}$mice. Weight loss appeared after $1-2 \mathrm{wk}$, accompanied by diarrhea and piloerection. At day 13, colitic mice were left untreated or received $1.4 \times 10^{6}$ Treg, purified from CD45.2 WT or $\mathrm{Tnfrsf}^{-1-}$ donors by magnetic separation as described above. Changes in body weight and clinical symptoms were monitored every 1-2 days. Serum was collected at day 37 upon colitis induction and TNF- $\alpha$ was measured by ELISA (eBioscience). Formalin-fixed, paraffin-embedded colon sections were stained with H\&E and colitis severity was graded according to a fourgrade semi-quantitative scoring system. T cells were obtained from mesenteric LN and lamina propria as previously described [26] and analyzed by flow cytometry.

\section{In vitro proliferation assay}

Purified $\mathrm{CD} 4{ }^{+} \mathrm{CD} 25^{+}$cells were labelled by incubation with $5 \mu \mathrm{M}$ CFSE (Invitrogen) in complete medium containing 10\% FBS for $15 \mathrm{~min}$ at $37^{\circ} \mathrm{C}$. A total of $5 \times 10^{4} \mathrm{CD} 4^{+} \mathrm{CD} 25^{+}$cells were cultured with $5 \times 10^{4}$ accessory cells (consisting of irradiated splenocytes) in the presence of $1 \mu \mathrm{g} / \mathrm{mL}$ of soluble anti-CD3 (e-Bioscience) in complete medium containing RPMI 1640 (Sigma-Aldrich) supplemented with 10\% FCS, 2 mM L-glutamine, $200 \mathrm{U}$ penicillin and $200 \mathrm{mg} / \mathrm{mL}$ streptomycin (Sigma-Aldrich). Where indicated, recombinant IL-2 (Proleukin) was added at scaled concentrations. Every condition was assayed in triplicate and analysed after $72 \mathrm{~h}$ by flow cytometry after gating on $\mathrm{CD} 4^{+}$ Foxp ${ }^{+}$cells.

\section{Western blot}

Fresh Treg, naïve and activated $\mathrm{CD}^{+} \mathrm{T}$ cells were lysed with a chilled lysis buffer (50 mM Hepes, pH 7.0, 0.1\% NP-40, $250 \mathrm{mM}$ $\mathrm{NaCl}, 1 \times$ Protease Inhibitor Cocktail). Cell debris was removed by centrifugation at $14000 \mathrm{rpm}$ for $20 \mathrm{~min}$ at $4^{\circ} \mathrm{C}$. Solubilized proteins were separated using SDS-PAGE and transferred to a PVDF membrane. SOCS1 and $\beta$-actin were visualized using the antibodies 4H1 (Invitrogen) and A2066 (Sigma), respectively. Densitometric analysis was performed using NIH Image $\mathrm{J}$ software.

\section{Real time RT-PCR}

Fifty nanograms of total RNA, isolated by using the mirVana miRNA isolation kit (Ambion), were subjected to reverse transcription according to the manufacturer's instructions (Applied Biosystems). Quantitative real time RT-PCR analysis for miR155 was performed according to the TaqMan MicroRNA Assays (Applied Biosystems) and samples normalized by evaluating RNU6 expression. Results were obtained using the comparative $\mathrm{Ct}$ method.

\section{Antibodies and flow cytometry}

PE-Cy7 anti-CD4 (RM4-5), FITC anti-CD8 (53-6.7), FITC antiCD25 (PC61), PE anti-OX40 (OX86), FITC anti-CD45.1 (A20), FITC anti-CD45.2 (104), PE anti-CD103 (2E7) were purchased from eBioscience. Surface staining was performed by incubating antibodies at $5 \mu \mathrm{g} / \mathrm{mL}$ on ice for $30 \mathrm{~min}$ in PBS containing $2 \%$ FBS. Intracellular staining with PE or APC anti-Foxp3 (FJK-16s) was performed according to manufacturer's instructions (eBioscience). For IL-10 and Foxp3 staining, cells obtained from LN and colonic lamina propria were stimulated with $50 \mathrm{ng} / \mathrm{mL}$ PMA, $500 \mathrm{ng} / \mathrm{mL}$ ionomycin (Sigma-Aldrich) with Monensin (eBioscience) for $4 \mathrm{~h}$ at $37^{\circ} \mathrm{C}$, fixed in Fix/Perm buffer (eBioscience) and stained with APC anti-Foxp3 and PE 
anti-IL-10 (JES5-16E3, eBioscience). To evaluate Stat5 phosphorylation, Treg were stimulated with rIL-2 (Proleukin) and stained with AlexaFluor488 anti-Stat5 pY694 (clone 47) according to the manufacturer's instructions (BD Bioscience). Flow cytometry data were acquired on a FACSCalibur (Becton Dickinson) and analysed with FlowJo software (version 8.8.4; Treestar).

\section{Statistical analysis}

Results are expressed as the means \pm SD. Data were analyzed using a nonpaired Student's $t$ test (Prism software, GraphPad).

Acknowledgements: This work was supported by grants from the Italian Ministry of Health (to M.P.C.) and AIRC (Associazione Italiana Ricerca sul Cancro) (to M.P.C. and S.P.). S.P. and P.P. are supported by fellowships from FIRC (Fondazione Italiana Ricerca sul Cancro). We thank Ivano Arioli and Chiara Ratti for technical assistance. We acknowledge Gabriella Abolafio and Andrea Vecchi for cell sorting.

Conflict of interest: The authors declare no financial or commercial conflict of interest.

\section{References}

1 Shevach, E. M., Mechanisms of foxp $3+\mathrm{T}$ regulatory cell-mediated suppression. Immunity 2009. 30: 636-645.

2 Takeda, I., Ine, S., Killeen, N., Ndhlovu, L. C., Murata, K., Satomi, S., Sugamura, K. and Ishii, N., Distinct roles for the OX40-OX40 ligand interaction in regulatory and nonregulatory T cells. J. Immunol. 2004. 172: 3580-3589.

3 Valzasina, B., Guiducci, C., Dislich, H., Killeen, N., Weinberg, A. D. and Colombo, M. P., Triggering of OX40 (CD134) on CD4(+)CD25+T cells blocks their inhibitory activity: a novel regulatory role for OX40 and its comparison with GITR. Blood 2005. 105: 2845-2851.

4 Sugamura, K., Ishii, N. and Weinberg, A. D., Therapeutic targeting of the effector T-cell co-stimulatory molecule OX40. Nat. Rev. Immunol. 2004. 4: $420-431$.

5 Rogers, P. R., Song, J., Gramaglia, I., Killeen, N. and Croft, M., OX40 promotes $\mathrm{Bcl}-\mathrm{xL}$ and $\mathrm{Bcl}-2$ expression and is essential for long-term survival of CD4 T cells. Immunity 2001. 15: 445-455.

6 Huddleston, C. A., Weinberg, A. D. and Parker, D. C., OX40 (CD134) engagement drives differentiation of $\mathrm{CD} 4+\mathrm{T}$ cells to effector cells. Eur. J. Immunol. 2006. 36: 1093-1103.

7 Bansal-Pakala, P., Jember, A. G. and Croft, M., Signaling through OX40 (CD134) breaks peripheral T-cell tolerance. Nat. Med. 2001. 7: 907-912.

8 So, T. and Croft, M., Cutting edge: OX40 inhibits TGF-beta- and antigendriven conversion of naive CD4 $\mathrm{T}$ cells into $\mathrm{CD} 25+$ Foxp3+T cells. J. Immunol. 2007. 179: 1427-1430.
9 Vu, M. D., Xiao, X., Gao, W., Degauque, N., Chen, M., Kroemer, A., Killeen, N. et al., OX40 costimulation turns off Foxp3+Tregs. Blood 2007. 110: 2501-2510.

10 Ito, T., Wang, Y. H., Duramad, O., Hanabuchi, S., Perng, O. A., Gilliet, M., Qin, F. X. and Liu, Y. J., OX40 ligand shuts down IL-10-producing regulatory T cells. Proc. Natl. Acad. Sci. USA 2006. 103: 13138-13143.

11 Piconese, S., Valzasina, B. and Colombo, M. P., OX40 triggering blocks suppression by regulatory $\mathrm{T}$ cells and facilitates tumor rejection. J. Exp. Med. 2008. 205: 825-839.

12 Hirschhorn-Cymerman, D., Rizzuto, G. A., Merghoub, T., Cohen, A. D., Avogadri, F., Lesokhin, A. M., Weinberg, A. D. et al., OX40 engagement and chemotherapy combination provides potent antitumor immunity with concomitant regulatory $\mathrm{T}$ cell apoptosis. J. Exp. Med. 2009. 206: 1103-1116.

13 Gri, G., Piconese, S., Frossi, B., Manfroi, V., Merluzzi, S., Tripodo, C., Viola, A. et al., CD4+CD25+regulatory T cells suppress mast cell degranulation and allergic responses through OX40-OX40L interaction. Immunity 2008. 29: 771-781.

14 Piconese, S., Gri, G., Tripodo, C., Musio, S., Gorzanelli, A., Frossi, B., Pedotti, R. et al., Mast cells counteract regulatory T-cell suppression through interleukin- 6 and OX40/OX40L axis toward Th17-cell differentiation. Blood 2009. 114: 2639-2648.

15 Maloy, K. J. and Powrie, F., Fueling regulation: IL-2 keeps CD4+Treg cells fit. Nat. Immunol. 2005. 6: 1071-1072.

16 Bayer, A. L., Jones, M., Chirinos, J., de Armas, L., Schreiber, T. H., Malek, T. R. and Levy, R. B., Host CD4+CD25+T cells can expand and comprise a major component of the Treg compartment after experimental HCT. Blood 2009. 113: 733-743.

17 Bayer, A. L., Yu, A. and Malek, T. R., Function of the IL-2R for thymic and peripheral $\mathrm{CD} 4+\mathrm{CD} 25+$ Foxp3 $+\mathrm{T}$ regulatory cells. J. Immunol. 2007. 178: 4062-4071.

18 Yao, Z., Kanno, Y., Kerenyi, M., Stephens, G., Durant, L., Watford, W. T., Laurence, A. et al., Nonredundant roles for Stat5a/b in directly regulating Foxp3. Blood 2007. 109: 4368-4375.

19 Lu, L. F., Thai, T. H., Calado, D. P., Chaudhry, A., Kubo, M., Tanaka, K., Loeb, G. B. et al., Foxp3-dependent microRNA155 confers competitive fitness to regulatory $\mathrm{T}$ cells by targeting SOCS1 protein. Immunity 2009. 30: 80-91.

20 Williams, C. A., Murray, S. E., Weinberg, A. D. and Parker, D. C., OX40mediated differentiation to effector function requires IL-2 receptor signaling but not CD28, CD40, IL-12Rbeta2, or T-bet. J. Immunol. 2007. 178: 7694-7702.

21 Almeida, A. R., Zaragoza, B. and Freitas, A. A., Competition controls the rate of transition between the peripheral pools of CD4+CD25- and CD4+ CD25+T cells. Int. Immunol. 2006. 18: 1607-1613.

22 Komatsu, N. and Hori, S., Full restoration of peripheral Foxp3+regulatory $\mathrm{T}$ cell pool by radioresistant host cells in scurfy bone marrow chimeras. Proc. Natl. Acad. Sci. USA 2007. 104: 8959-8964.

23 Scott-Browne, J. P., Shafiani, S., Tucker-Heard, G., Ishida-Tsubota, K., Fontenot, J. D., Rudensky, A. Y., Bevan, M. J. and Urdahl, K. B., Expansion and function of Foxp3-expressing $\mathrm{T}$ regulatory cells during tuberculosis. J. Exp. Med. 2007. 204: 2159-2169.

24 Izcue, A., Coombes, J. L. and Powrie, F., Regulatory lymphocytes and intestinal inflammation. Annu. Rev. Immunol. 2009. 27: 313-338.

25 Mottet, C., Uhlig, H. H. and Powrie, F., Cutting edge: cure of colitis by CD4+CD25+regulatory T cells. J. Immunol. 2003. 170: 3939-3943.

26 Uhlig, H. H., Coombes, J., Mottet, C., Izcue, A., Thompson, C., Fanger, A., Tannapfel, A. et al., Characterization of Foxp3+CD $4+C D 25+$ and 
IL-10-secreting CD4+CD25+T cells during cure of colitis. J. Immunol. 2006. 177: 5852-5860.

27 Haribhai, D., Lin, W., Edwards, B., Ziegelbauer, J., Salzman, N. H., Carlson, M. R., Li, S. H. et al., A central role for induced regulatory T cells in tolerance induction in experimental colitis. J. Immunol. 2009. 182: 3461-3468.

28 Annacker, O., Coombes, J. L., Malmstrom, V., Uhlig, H. H., Bourne, T., Johansson-Lindbom, B., Agace, W. W. et al., Essential role for CD103 in the $\mathrm{T}$ cell-mediated regulation of experimental colitis. J. Exp. Med. 2005. 202: 1051-1061.

29 Banz, A., Peixoto, A., Pontoux, C., Cordier, C., Rocha, B. and Papiernik, M., A unique subpopulation of CD4+regulatory $\mathrm{T}$ cells controls wasting disease, IL-10 secretion and T cell homeostasis. Eur. J. Immunol. 2003. 33: 2419-2428.

30 Malek, T. R. and Bayer, A. L., Tolerance, not immunity, crucially depends on IL-2. Nat. Rev. Immunol. 2004. 4: 665-674.

31 Thornton, A. M., Donovan, E. E., Piccirillo, C. A. and Shevach, E. M., Cutting edge: IL-2 is critically required for the in vitro activation of CD4+ CD25+T cell suppressor function. J. Immunol. 2004. 172: 6519-6523.

32 Sporri, B., Kovanen, P. E., Sasaki, A., Yoshimura, A. and Leonard, W. J., JAB/SOCS1/SSI-1 is an interleukin-2-induced inhibitor of IL-2 signaling. Blood 2001. 97: 221-226.

33 So, T., Lee, S. W. and Croft, M., Immune regulation and control of regulatory T cells by OX40 and 4-1BB. Cytokine Growth Factor Rev. 2008. 19: 253-262.

34 Gounaris, E., Blatner, N. R., Dennis, K., Magnusson, F., Gurish, M. F., Strom, T. B., Beckhove, P. et al., T-regulatory cells shift from a protective anti-inflammatory to a cancer-promoting proinflammatory phenotype in polyposis. Cancer Res. 2009. 69: 5490-5497.

35 Liu, H., Hu, B., Xu, D. and Liew, F. Y., CD4+CD25+regulatory T cells cure murine colitis: the role of IL-10, TGF-beta, and CTLA4. J. Immunol. 2003. 171: 5012-5017.

36 Rubtsov, Y. P., Rasmussen, J. P., Chi, E. Y., Fontenot, J., Castelli, L., Ye, X., Treuting, P. et al., Regulatory $\mathrm{T}$ cell-derived interleukin-10 limits inflammation at environmental interfaces. Immunity 2008. 28: 546-558.

37 Francisco, L. M., Salinas, V. H., Brown, K. E., Vanguri, V. K., Freeman, G. J., Kuchroo, V. K. and Sharpe, A. H., PD-L1 regulates the development, maintenance, and function of induced regulatory T cells. J. Exp. Med. 2009. 206: 3015-3029.

38 Schmidt, E. M., Wang, C. J., Ryan, G. A., Clough, L. E., Qureshi, O. S., Goodall, M., Abbas, A. K. et al., Ctla-4 controls regulatory T cell peripheral homeostasis and is required for suppression of pancreatic islet autoimmunity. J. Immunol. 2009. 182: 274-282.
39 Malmstrom, V., Shipton, D., Singh, B., Al-Shamkhani, A., Puklavec, M. J., Barclay, A. N. and Powrie, F., CD134L expression on dendritic cells in the mesenteric lymph nodes drives colitis in T cell-restored SCID mice. J. Immunol. 2001. 166: 6972-6981.

40 Totsuka, T., Kanai, T., Uraushihara, K., Iiyama, R., Yamazaki, M., Akiba, H., Yagita, H. et al., Therapeutic effect of anti-OX40L and anti-TNF-alpha MAbs in a murine model of chronic colitis. Am. J. Physiol. Gastrointest. Liver Physiol. 2003. 284: G595-G603.

41 Obermeier, F., Schwarz, H., Dunger, N., Strauch, U. G., Grunwald, N., Scholmerich, J. and Falk, W., OX40/OX40L interaction induces the expression of CXCR5 and contributes to chronic colitis induced by dextran sulfate sodium in mice. Eur. J. Immunol. 2003. 33: 3265-3274.

42 Klinger, M., Kim, J. K., Chmura, S. A., Barczak, A., Erle, D. J. and Killeen, N., Thymic OX40 expression discriminates cells undergoing strong responses to selection ligands. J. Immunol. 2009. 182: 4581-4589.

43 Kroemer, A., Xiao, X., Vu, M. D., Gao, W., Minamimura, K., Chen, M., Maki, T. and Li, X. C., OX40 controls functionally different $\mathrm{T}$ cell subsets and their resistance to depletion therapy. J. Immunol. 2007. 179: 5584-5591.

44 Kohm, A. P., McMahon, J. S., Podojil, J. R., Begolka, W. S., DeGutes, M., Kasprowicz, D. J., Ziegler, S. F. and Miller, S. D., Cutting Edge: Anti-CD25 monoclonal antibody injection results in the functional inactivation, not depletion, of $\mathrm{CD} 4+\mathrm{CD} 25+\mathrm{T}$ regulatory cells. J. Immunol. 2006. 176: 3301-3305.

45 Kryczek, I., Wei, S., Zou, L., Altuwaijri, S., Szeliga, W., Kolls, J., Chang, A. and Zou, W., Cutting edge: Th17 and regulatory T cell dynamics and the regulation by IL-2 in the tumor microenvironment. J. Immunol. 2007. 178: 6730-6733.

46 Laurence, A., Tato, C. M., Davidson, T. S., Kanno, Y., Chen, Z., Yao, Z., Blank, R. B. et al., Interleukin-2 signaling via STAT5 constrains T helper 17 cell generation. Immunity 2007. 26: 371-381.

Full correspondence: Dr. Mario P. Colombo, Fondazione IRCCS Istituto Nazionale dei Tumori, via Amadeo 42, 20133 Milan, Italy

Fax: +39-0223902630

e-mail: mario.colombo@istitutotumori.mi.it

Received: $18 / 3 / 2010$

Revised: $20 / 6 / 2010$

Accepted: 13/7/2010

Article accepted online: 26/7/2010 